\title{
Intrusion Detection using Machine Learning
}

\author{
Lavanya.P, Sangeetha.A, Santhana Krishnan
}

\begin{abstract}
System savage technicians work to keep administrations accessible every time by dealing with gatecrasher assaults. Interruption Recognition System (IRS) is one of the possible components that is used to detect and order any anomalous activities. In this manner, the IRS must be dependably fully informed regarding the most recent gatecrasher assaults marks to save privacy, trustworthiness, and accessibility of administrations. The fast of IRS is an imperative problem. This examination work represents how the Knowledge Disclosure and Data Mining (or Knowledge Discovery in Databases) The CART and RBFN have been picked for this investigation. It has been demonstrated that the CART classifier has accomplished the most elevated exactness rate for distinguishing and arranging all $\mathrm{KDD}$ dataset assaults, which are of sort DOS, R2C, C2R, and Test.
\end{abstract}

Index Terms: Classification and Regression Trees, Interruption Recognition system Knowledge Discovery in Database, Radical basis Function network.

\section{INTRODUCTION}

Managing a dependable system is troublesome errand thinking about all unique conceivable kinds of assaults. These days, PC systems and their administrations are generally utilized in industry, business, and all fields of life. Security work force and everybody who has an obligation regarding giving insurance for a system and its clients, have genuine concerns about gatecrasher assaults. System chairmen and security officers attempt to give an ensured situation for client's records, organize assets, individual records and passwords. Aggressors may carry on in two different ways to do their assaults on systems; one of these ways is to make a system administration inaccessible for clients or on the other hand damaging individual data. Denial of Service (DoS) is a standout amongst the most incessant cases speaking to assaults on system assets and making system administrations inaccessible for their clients. There are numerous sorts of DoS assaults, and each sort has it is possess conduct on expending system assets to accomplish the gatecrasher's point, which is to render the system inaccessible for its clients. Remote to client (R2C) is one sort of PC arrange assaults, in which a gatecrasher sends set of bundles to another PC or server over a system where he/she doesn't have consent to access as a neighborhood client. Client to root assaults (C2R)

is a second sort of assault where the interloper endeavors to get to the system assets as a typical client, furthermore, after a few endeavors, the interloper progresses toward becoming as

Revised Manuscript Received on July 22, 2019.

Lavanya.P, M.E (CSE with networks), , Department of IT, PSNACET, Dindigul-624622, India Email id :lavi.shivam22@ gmail.com

Sangeetha.A, M.E Assistant Professor, Department of IT, PSNACET, Dindigul-624622, India Email id: sangeetha@psnacet.edu.in,

Santhana Krishnan, M.E Assistant Professor, Department of ECE, SCADCET, Tirunelveli ,India Email id:santhanakrishnan86@gmail.com a full get to client.[1]Testing is a third sort of assault in which the interloper filters organize gadgets to decide short coming in topology plan or some opened ports and after that utilization them in the future for unlawful access to individual data. There are numerous models that speak to testing over a system, for example, nmap, portsweep, ipsweep. IRS turns into a basic part to fabricate PC system to catch these sorts of assaults in beginning periods, since IRS neutralizes all gatecrasher assaults. IDS employments characterization systems to settle on choice about each parcel go through the system whether it is an ordinary parcel or an assault (for example DOS, C2R, R2C, PROBE) parcel. KDD is an online vault dataset, which incorporates all sorts of gatecrashers', assaults, for example, DOS, R2C, C2R, and Test.[2] In this paper, various classifiers will be assessed on the KDD dataset. The technique followed in this investigation is first to play out a preprocessing venture on KDD dataset and after to utilize the readied dataset on a reasonable condition and assets, lastly, to look at which classifier is more precise than others in recognizing all contemplated assaults (DOS, R2C, C2R).The rest of this paper deeply explained about machine learning in section II. In section III defines the detail description in cryptography.Comparsion of machine learning and cryptography comes up in section IV. Final section deals with proposed system in CART and RBFN.

\section{II.MACHINE LEARNING}

A. Definition

Machine Learning is a subset of computerized reasoning which centers chiefly around machine learning from their experience and making expectations dependent on its experience. It empowers the PCs or the machines to settle on information driven choices as opposed to being expressly modified for doing a specific undertaking. These projects or calculations are planned such that they learn and enhance after some time when are presented to new information.

\section{B. Types of Machine Learning:}

Supervised Learning:

Supervised learning is a learning in which we instruct or train the machine utilizing information which is very much named that implies a few information is as of now labeled with right answer. A perfect circumstance will consider the computation to viably choose the class marks for disguised cases. It is classified into two types of algorithm: Classification and Regression. In Classification, the output variable is category.

\section{Unsupervised Learning:}

Unsupervised learning is the preparation of machine utilizing data that is neither ordered nor named and

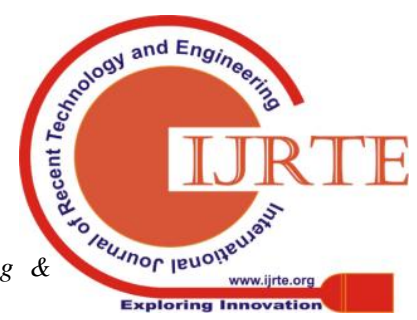


enabling the calculation to follow up on that data without direction. It distinguishes shared traits in the information and responds dependent on the nearness or nonattendance of such shared characteristics in each new bit of information. It is alternative to supervised learning.It is classified into two types of algorithm: Clustering and Association

\section{Reinforcement Learning:}

It lies between supervised and unsupervised learning .This algorithm defines the answer as wrong for particular target example taken and it does not define how to correct it. In order to correct this, try different possibilities until the correct answer is drawn.

\section{Evolutionary Learning:}

It is a simple genetic algorithm (SGA) and comes with genetic programming. It is also called as biological learning. It is to improve their survival rates and details of offspring of biological organisms.[3]

\section{Cryptography:}

Cryptography modifies over data into a design that is indiscernible for an unapproved customer, empowering it to be transmitted without unapproved components translating it by and by into a noticeable course of action, in this manner exchanging off the data. Information security uses cryptography on a couple of measurements. The information can't be scrutinized without a key to unscramble it. The information keeps up its trustworthiness in the midst of movement and remembering that being secured. Cryptography similarly helps in non denial. This infers the sender and the movement of a message can be affirmed. It is otherwise called cryptology. There are three kinds of calculation. They are Secret Key Cryptography (SKC), Public Key Cryptography (PKC) and Hash capacities.

\section{A.Types}

\section{Secret key Cryptography:}

Secret key cryptography techniques utilize a key for encoding and decoding. The sender uses the best approach to encode the plaintext and sends the figure content to the gatherer. The recipient applies a comparative key to unscramble the message and recover the plaintext. Since a single key is used for the two limits, mystery key cryptography is moreover called symmetric encryption. With this kind of cryptography, obviously the key must be known to both the sender and the gatherer; that, without a doubt, is the puzzle. The best issue with this philosophy, clearly, is the transport of the key (more on that later in the talk of open key cryptography).

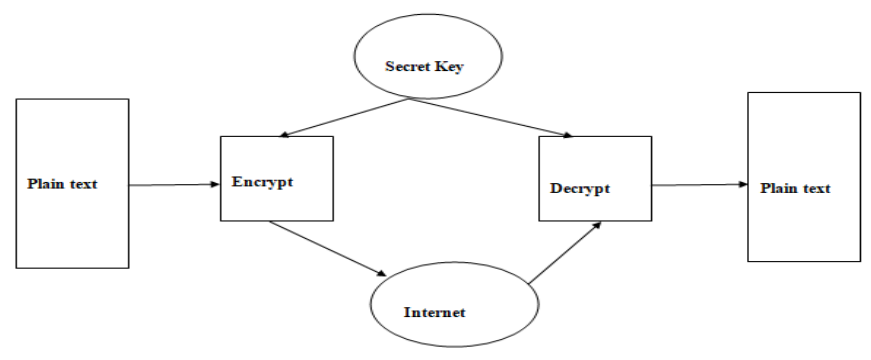

Fig 1: Secret Key Cryptography

\section{Public Key Cryptography:}

Diverse keys are utilized for encryption and decoding process. PKC uses two keys that are numerically related regardless of the way that learning of one key does not empower someone to viably choose the other key. One key is used to encode the plaintext and the other key is used to interpret the figure content. Use of pair of keys is said to be hilter kilter cryptography. In PKC, one of the keys is doled out individuals in open key and may be advertised as extensively as the owner needs. The other key is allocated the private key and is never revealed to another social affair. It is straight-forward to send messages under this arrangement. It is used for authentication, nonrepudiation, key exchange. Some of PKC are RSA, Diffe-Hellman, Digital Signature algorithm etc.

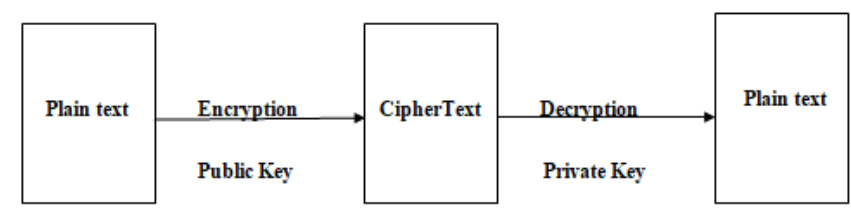

Fig 2: Public key Cryptography

\section{Hash Functions:}

The hash functions do not use keys because it has fixed-length hash value which is dependent on plain text. It is also called message digest. Hash algorithms are normally used to give a propelled interesting finger impression of a report's substance routinely used to ensure that the record has not been altered by a gatecrasher or infection. Hash capacities are furthermore normally used by many working structures to scramble passwords. Hash capacities, by then, give a framework to ensure the decency of a report. Commonly used hash algorithms are Message Digest (MD), Secure Hash Algorithms (SHA) etc.

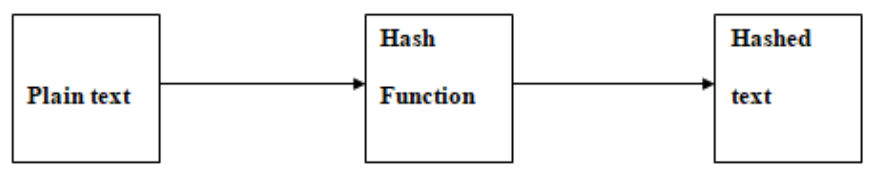

Fig 3: Hash Function

\section{MACHINE LEARNING AND CRYPTOGRAPHY}

AI(ML) and cryptanalysis can be viewed as sister fields," since they share various of comparative thoughts and concerns. In an average cryptanalytic situation, the cryptanalysts wishes to "break" some cryptosystem. Ordinarily this suggests he wishes to find the mystery key used by the customers of the cryptosystem, where the general system is starting at now known. The unscrambling limit along these lines starts from a known gathering of such limits (requested by the key), and the target of the cryptanalyst is to decisively separate which such limit is being used. He may ordinarily have available a tremendous measure of planning figure content and plaintext to use in his assessment. This issue can similarly be portrayed as the issue of "learning a dark capacity". [4] 


\section{V.COMPARISON OF CRYPTOGRAPHY AND MACHINE LEARNING}

\begin{tabular}{|l|l|}
\hline Cryptography & Machine Learning \\
\hline $\begin{array}{l}\text { It has secret key and key space. All these } \\
\text { keys are used for encryption process }\end{array}$ & $\begin{array}{l}\text { It has target function and class of } \\
\text { possible target functions. But it does not } \\
\text { need that process. }\end{array}$ \\
\hline $\begin{array}{l}\text { The complexity of undefined key is } \\
\text { known as cryptanalyst. Later, the } \\
\text { technique called variable-fixed length is } \\
\text { used }\end{array}$ & $\begin{array}{l}\text { The size of the target is unknown before } \\
\text { the target starts to work. The method } \\
\text { called complexity of hypothesis is used. }\end{array}$ \\
\hline $\begin{array}{l}\text { Exact Inference is possible } \\
\text { Approximate Inference is not possible }\end{array}$ & $\begin{array}{l}\text { Exact Inference is difficult to perform } \\
\text { Approximate Inferenceis easy to achieve }\end{array}$ \\
\hline $\begin{array}{l}\text { Computational complexity of } \\
\text { cryptography is showing weakest } \\
\text { possible complexity assumptions } \\
\text { Polynomial-time algorithm does not } \\
\text { provide security. }\end{array}$ & $\begin{array}{l}\text { Computational complexity of machine } \\
\text { leaming is finding hypothesis is } \\
\text { consistent. The achievement of } \\
\text { polynomial time leaming algorithm } \\
\text { provides efficient complexity to target } \\
\text { examples taken. }\end{array}$ \\
\hline $\begin{array}{l}\text { Unicity Distance=M(N)/D } \\
\text { Where M(N) is entropy of key space N } \\
\text { and D is redundancy language }\end{array}$ & $\begin{array}{l}\text { Unicity Distance of cryptography is } \\
\text { equal to unicity diatance of machine } \\
\text { leaming. }\end{array}$ \\
\hline $\begin{array}{l}\text { The unicity distance is an "information } \\
\text { theoretic" proportion of the measure of } \\
\text { information that a cryptanalyst needs to } \\
\text { prevail in recognizing the mystery key. }\end{array}$ & $\begin{array}{l}\text { leaming plays a same role as } \\
\text { cryptography. }\end{array}$ \\
\hline
\end{tabular}

\section{VI.CRYPTOGRAPHY IMPACTS ON LEARNING THEORY:}

The irregular capacities suggests that even around learning the class of capacities represent able by polynomial-size boolean circuits is infeasible, expecting that single direction capacities exist, regardless of whether the student is permitted to question the obscure capacity. So specialists in AI have focused on the subject of distinguishing which less complex classes of capacities are learnable (around, from irregular models, or precisely, with questions). For instance, a noteworthy open inquiry in the field is whether the class of boolean capacities represent able as boolean equations in disjunctive typical structure (DNF) is productively learnable from irregular precedents. The essential effect of cryptography on AI hypothesis is a characteristic (yet negative) one: appearing certain learning issues are computationally recalcitrant. There are different manners by which a learning issue could be recalcitrant; for instance, learning the class of every boolean capacity is immovable just on the grounds that the required number of precedents is exponentially expansive in the quantity of boolean information factors. A specific class of recalcitrance results for learning hypothesis is portrayal subordinate: they demonstrate that given a lot of models, finding a steady boolean capacity spoke to with a specific goal in mind is computationally obstinate. For instance, finding a 2-term DNF recipe steady with a lot of information/yield sets for such an objective equation is a NP-complete issue. This infers learning 2-term DNF is recalcitrant (accepting P r NP), yet just if the student is required to deliver his answer as a 2-term DNF equation. The relating issue for capacities represent able in 2 (CNF with two literals for every proviso, which legitimately contains the set of capacities represent able in 2-term DNF) is tractable, thus PAC-! procuring the class of capacities represent able in 2-term DNF is conceivable, as long as the student may yield his answers in 2-CNF. So, they demonstrated that it is NP-finished to discover a base size DFA that is steady with a given arrangement of
Information/yield precedents, and have stretched out this outcome to demonstrate that finding an approximately least size DFA predictable with such a lot of models is difficult to do productively. These portrayal subordinate outcomes rely upon the supposition that $\mathrm{P} \sim \mathrm{NP}$. So as to acquire hardness results that are portrayal autonomous, the swung to cryptographic suppositions (to be specific, the trouble of modifying RSA, the trouble of perceiving quadratic buildups modulo a Blum whole number, and the trouble of figuring Blum whole numbers). Obviously, they likewise need to clarify how learning could be hard in a portrayal free way, which they do by requiring the learning calculation not to yield a speculation in some portrayal language, but instead to foresee the characterization of another model with high precision.

\section{VII.MACHINE LEARNING IMPACT ON CRYPTOGRAPHY}

Since the vast majority of the negative outcomes in learning hypothesis as of now rely upon cryptographic suspicions, there has been no effect of negative outcomes on learning hypothesis on the advancement of cryptographic plans. Maybe a portion of the outcomes and ideas and It could be connected toward this path, yet this has not been finished. Then again, the positive outcomes in learning hypothesis are ordinarily free of cryptographic suppositions, and could on a basic level be

connected to the cryptanalysis of generally straightforward cryptosystems. Quite a bit of this dialog will be theoretical in nature, since there is little in the writing investigating these conceivable outcomes. We sketch some conceivable approaches, yet leave their closer examination and approval(either hypothetical or experimental) as open issues.[5]

\section{VIII.LITERATURE SURVEY}

Mouhammad Alkasassbeh, Mohammad Almseidin,[6] proposed a detection system called"Machine Learning Methods for Network Intrusion Detection". Framework savage engineers work to keep organizations open available every time by dealing with interloper strikes. Interruption Detection System (IDS) is one of the reachable instruments is utilized to distinguish and describe more unpredictable exercises. Thusly, the IDS must be constantly completely educated with respect to the latest intruder attacks imprints to defend order, uprightness, and availability of the administrations. The fast of the IDS is a fundamental issue . This investigation work indicates how the KDD dataset is incredibly advantageous for testing and surveying remarkable AI Techniques. It generally revolves around the KDD preprocess part to set up a not that entire awful and sensible preliminary enlightening file. The MLP and Bayes Network classifiers have been picked for this examination. It has been shown that the classifier has achieved the most significant precision rate for recognizing and describing all KDD dataset strikes, which are of sort DOS, R2L, U2R, and Test.

Suad Mohammed Othman, Fadl Mutaher Ba- Alwi, Nabeel T. Alsohybe1 and Amal Y.Al- Hashida, defined a intrusion detection system based on machine learning in big data called"Intrusion detection 
model using machine learning algorithm on Big Data environment"[7] The enormous proportions of data and its continuous addition will modified the centrality of information security and data examination structures for Big Data. Interruption Detection System (IDS) is a structure it screens, separates data to recognize any interruption in the system or framework. Huge volume, arrangement and quick of data made in the framework have made the data examination method to recognize attacks by standard procedures extraordinarily troublesome. Huge Data frameworks are used in IDS to oversee Big Data for precise and beneficial data examination process. This paper exhibited Spark-Chi-SVM appear for interruption recognition. In this model, we have used Chi Sq Selector for feature assurance, and manufactured an interference acknowledgment show by using reinforce vector machine (SVM) classifier on Apache Spark Big Data organize. We used KDD99 to get ready and test the model. In the test, we introduced a relationship between's Chi-SVM classifier what's more, Chi-Logistic Regression classifier. The eventual outcomes of the preliminary exhibited that Spark-Chi-SVM show has first class, diminishes the planning time and is gainful for huge Information.

Hang $\mathrm{Xu}$ and Frank Mueller, Mithun Acharya and Alok Kucheria[8]defined a system called"Machine Learning Enhanced Real-Time Intrusion Detection Using Timing Information" The examined interruption recognition systems for ongoing control gadgets. This work contributes a novel structure of isolating security checking and location from ongoing control, where the previous is performed on Cloud edge gadgets while the last is kept running on inserted gadgets connected to the framework that is controlled. We contribute a security checking framework that approves most pessimistic scenario timing limits of the objective controller and furthermore approves its control

yields by contrasting it against model-based expectations, which are determined from AI.

Nutan Farah Haq ,Abdur Rahman Onik ,Md. Avishek Khan Hridoy ,Musharrat Rafni ,Faisal

Muhammad Shah Dewan Md. Farid [9]given a survey named "Application of Machine Learning Approaches in Intrusion Detection System: A Survey". System security is one of the significant worries of the cutting edge time. With the quick improvement and huge utilization of web over the earlier decade, the vulnerabilities of framework security have transformed into a basic issue.Interruption discovery framework is utilized to distinguish unapproved get to and unordinary assaults over the verified systems. Over the previous years, numerous investigations have been led on the interruption location framework.

Anna L. Buczak, Erhan Guven proposed a detail survey called "A Survey of Data Mining and Machine Learning Methods for Cyber Security Intrusion Detection",[10 ] This overview paper portrays an engaged writing overview of $\mathrm{AI}(\mathrm{ML})$ and information mining (DM) techniques for digital examination in help of interruption identification. Short instructional exercise portrayals of every
ML/DM technique are given. In light of the quantity of references or the significance of a rising technique, papers speaking to every technique were recognized, perused, and condensed. Since information is so vital in ML/DM approaches, some notable digital informational indexes utilized in ML/DM are depicted. The unpredictability of ML/DM calculations is tended to, dialog of difficulties for utilizing ML/DM for digital security is introduced, and what's more, a few suggestions on when to utilize a given strategy are given.

\section{CART:}

\section{PROPOSED SYSTEM}

It represents Classification and Regression Trees. It was presented by Breiman in 1984. The classification tree is built via CART by the twofold part of the characteristic. Gini Index is utilized as choosing the part quality. The CART is additionally utilized for relapse investigation with the assistance of relapse tree. The relapse highlight of CART can be utilized in guaging a needy variable given a lot of indicator variable over a given timeframe. CART has a normal speed of preparing and backings both constant and ostensible quality information.

\section{Advantages of CART:}

CART can deal with missing qualities naturally utilizing surrogate parts.

Uses any blend of consistent/discrete factors.

CART consequently performs variable choice.

CART can set up communications among factors

CART does not fluctuate as indicated by the monotonic change of prescient variable

\section{Multilayer Preceptron: \\ Radial Basis Function Network(RBFN):}

A RBFN is a particular sort of neural framework. In this article, I'll be depicting it's use as a non-straight classifier. All things considered, when people talk about neural frameworks or

"Counterfeit Neural Networks" they are implying the Multilayer Perceptron (MLP). Each neuron in a MLP takes the weighted entire of its data regards. That is, every data regard is copied by a coefficient, and the results are by and large summed together. A singular MLP neuron is a clear immediate classifier, yet complex non-direct classifiers can be worked by joining these neurons into a framework. A RBFN performs request by evaluating the data's closeness to points of reference from the arrangement set. Each RBFN neuron stores a "demonstrate", which is just a single of the points of reference from the planning set. When we have to arrange another data, each neuron figures the Euclidean division between the data and its model. For the most part, if the information all the more eagerly takes after the class A models than the class B models, it is designated class A. 


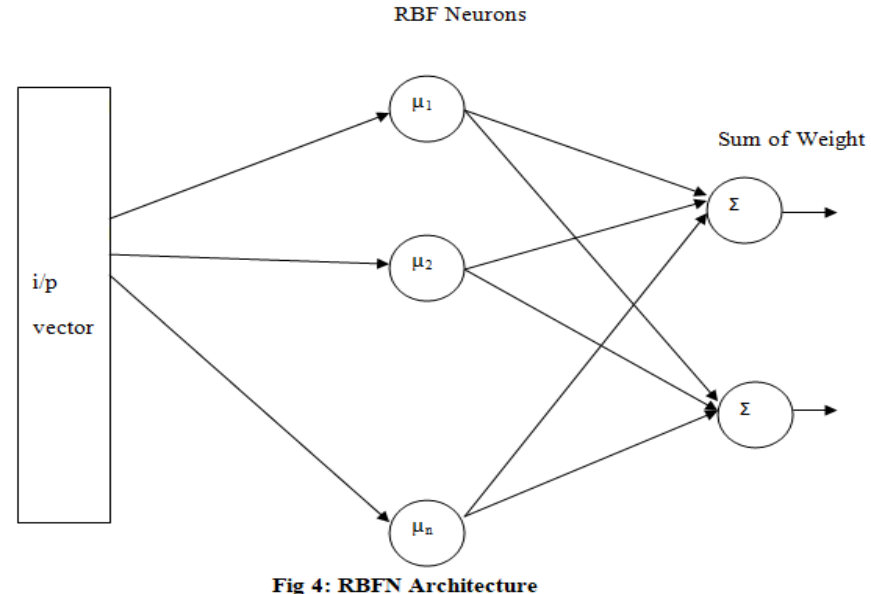

The above portrayal shows the customary designing of a RBF Network. It includes an information vector(i/p), a layer of $\mathrm{RBF}$ neurons, and a yield layer(o/p) with one center for each characterization or class of data.

\section{Information vector $(\mathbf{i} / \mathbf{p})$ :}

The information vector is the n-dimensional vector that you are endeavoring to describe. The entire information vector is seemed all of the RBF neurons.

\section{The RBF Neurons :}

Every RBF neuron stores a "show" vector which is just a single of the vectors from the readiness set. Each RBF neuron takes a gander at the information vector to its model, and yield a motivator some place in the scope of 0 and 1 which is an extent of comparability. If the information is identical to the model, by then the yield of that RBF neuron will be 1 . As the division between the information and model builds up, the response tumbles off exponentially towards 0 . The condition of the RBF neuron's response is a ringer twist, as illustrated in the framework configuration diagram. The neuron's response regard is moreover called its "activation" regard. The model vector is similarly consistently called the neuron's "inside", since it's the impetus at the point of convergence of the ring twist.

\section{Yield vector :}

The yield of the framework includes a great deal of centers, one for every order that we are trying to portray. Each yield center procedures a sort of score for the related class. Ordinarily, a request decision is made by consigning the commitment to the characterization with the most shocking score. The score is prepared by taking a weighted aggregate of the institution regards from each RBF neuron. By weighted absolute we infer that a yield center point relates a weight a motivating force with all of the RBF neurons, and expands the neuron's institution by this weight before adding it to the hard and fast response.

Since each yield center point is preparing the score for a substitute grouping, each yield center has its very own course of action of burdens. The yield center point will normally give a positive burden to the RBF neurons that have a spot with its class, and a negative burden to the others.

\section{X.CONCLUSION}

Because of the pressing interest for a viable IDS in system security, scientists are endeavoring to recognize improved approaches. This work outlines how the KDD dataset is very helpful for testing distinctive classifiers. The work concentrates on KDD preprocess stage to get ready reasonable trials and completely randomized autonomous test information. Among the arrangement methods (CART and RBFN), the CART classifier has accomplished the most astounding precision

rate for recognizing and classifying. The future work more classifiers will be tried just as the element determination to see the most essential highlights.

\section{REFERENCES:}

[1] Nicolas Papernot, Patrick McDaniel, Arunesh Sinha, and Michael Wellman,"SoK: Towards the Science of Security and Privacy in Machine Learning"Published in arxiv,Nov

2016.https://pdfs.semanticscholar.org/ebab/687cd1be7d25392c 11f89fce6a63bef7219d.pdf

[2] Marco Barreno · Blaine Nelson - Anthony D. Joseph .J.D Tygar,"The security of machine learning"published in springer link,april 2006. DOI 10.1007/s10994-010-5188-5

[3] wang hua,MA cuiqin,ZHOU Lijuan," A Brief Review of Machine Learning and its Application ", published in IEEE,2009

DOI: $10.1109 /$ ICIECS.2009.5362936

[4] Yohei Okada*, Shingo Ata*, Nobuyuki Nakamura $\not$, Yoshihiro Nakahira $\%$, and Ikuo Oka*"Comparisons of Machine Learning Algorithms for Application Identification of Encrypted Traffic",published in International conference on machine learning and application._DOI: $10.1109 /$ ICMLA.2011.162

[5] Ronald L. Rivest “Cryptography and Machine Learning", published in springer, 2015 https://link.springer.com/book/10.1007\%2F978-3-319-94147-9

[6] Mouhammad Alkasassbeh, Mohammad Almseidin, Machine Learning Methods for Network Intrusion Detection, published in IEEEpublications, 2016

https://www.researchgate.net/publication/327550168 Machine Learning_Methods_for_Network_Intrusion_Detection

[7] Suad Mohammed Othman1*, Fadl Mutaher Ba- Alwi1, Nabeel T. Alsohybe1 and Amal Y. Al- Hashida,Intrusion detection model using machine learning algorithm on Big Data environment,published in open access research,2017https://doi.org/10.1186/s40537-018-0145-4

[8] Hang Xu and Frank Mueller, Mithun Acharya and Alok Kucheria, Machine Learning Enhanced Real-Time Intrusion Detection Using Timing Information,published in IEEE ,2012. https://arcb.csc.ncsu.edu/ mueller/ftp/pub/mueller/papers/trec $4 \mathrm{c}$ ps18.pdf

[9] Nutan Farah Haq, Musharrat Rafni, Abdur Rahman Onik, Faisal Muhammad Shah, Md. Avishek Khan Hridoy, Application of Machine Learning Approaches in Intrusion Detection System: A Survey,published in International Journal of Advanced Research in Artificial Intelligence, Vol. 4, No.3, 2015,

https://thesai.org/Downloads/IJARAI/Volume4No3/Paper_2-Ap plication_of_Machine_Learning_Approaches_in_Intrusion_Det ection_System.pdf

[10] Anna L. Buczak, Erhan Guven[mach intrusion detection system] proposed a detail survey called "A Survey

of Data Mining and Machine Learning Methods for Cyber Security Intrusion Detection",published in IEEE explorer,2014

DOI: $10.1109 /$ COMST.2015.2494502 


\section{AUTHORS PROFILE}

P.Lavanya was born in Tamilnadu, India in 1994. She pursing M.E in the area of Computer Science and Engineering specialization in networks and B.Tech in the area of Information Technology from Anna University, India during 2012 and 2016 respectively. She completed her PG and UG at PSNA College of Engineering and Technology, Dindigul, TamilNadu, India. Her area of interest includes Machine learning, 1 11. $\begin{aligned} & \text { area of interest includes Machine learning, } \\ & \text { network security, internet of things, wireless }\end{aligned}$ sensor networks. She has a year of industrial experience in the department of Information Technology. She has published 3 research papers in international journals and presented papers in national and international conferences.

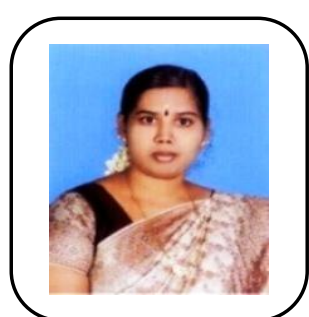

A.Sangeetha was born in Tamilnadu, India in 1983. She received M.E in the area of Computer Science and Engineering and B.Tech in the area of Information Technology from Anna University, India during 2005 and 2008 respectively. Currently she is working as a Assistant Professor in the department of Information Technology at PSNA College of Engineering and Technology, Dindigul, TamilNadu, India. Her area of interest includes mobile ad hoc networks and network security, internet of things, wireless sensor networks. She has 11 years of teaching experience in the department of Information Technology. She has published 8 research papers in international journals and presented more than 5 papers in national and international conferences.

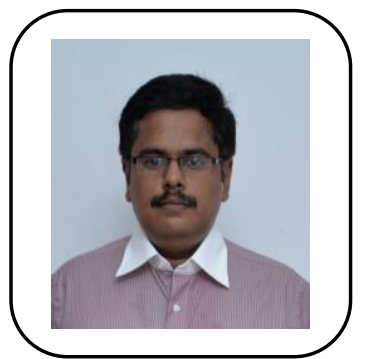

R.Santhana Krishnan was born in Tamilnadu, India in 1986. He received M.E in the area of Embedded System and B.E in the area of Electronic Communication Engineering from Anna University, India during 2008 and 2014 respectively. Currently he is working as a Assistant Professor in the department of ECE at SCAD College of Engineering and Technology, Tiruneveli, TamilNadu, India. His area of interest includes mobile ad hoc networks, embedded System wireless sensor networks. He has 7 years of teaching experience in the department of Information Technology. He has published 6 research papers in international journals and presented more than 3 papers in national and international conferences. 